\title{
Health assessment of trees using GPR-derived root density maps
}

\author{
Livia Lantini", Fabio Tosti ${ }^{*}$, Iraklis Giannakis", Lilong Zou*, Daniel Egyir ${ }^{*}$, Dale Mortimer ${ }^{\dagger}$, and Amir M. Alani ${ }^{*}$ \\ *School of Computing and Engineering, University of West London (UWL), St Mary's Road, Ealing, London W5 5RF, UK \\ ${ }^{\dagger}$ Tree Service, London Borough of Ealing, Perceval House, 14-16 Uxbridge Road, Ealing, London W5 2HL, UK
}

Email: Livia.Lantini@uwl.ac.uk; Fabio.Tosti@uwl.ac.uk; Iraklis.Giannakis@uwl.ac.uk; Lilong.Zou@uwl.ac.uk; Daniel.Egyir@uwl.ac.uk; MortimerD@ealing.gov.uk; Amir.Alani@uwl.ac.uk

\begin{abstract}
In this paper, a coherent framework for estimating the density and the distribution of roots using ground penetrating radar is presented. The proposed methodology is a multi-stage data processing scheme that is applied in semi-circular measurements collected concentrically around the investigated tree. The adopted processing methodology consists of three distinct and sequential steps. In the first step, the raw B-scans are subject to time-zero correction, zero-offset removal, time-varying gain and the singular value decomposition (SVD) filter. The SVD filter is used in order to effectively eliminate multiples and ringing noise from the B-Scans and increase the overall signal-to-clutter ratio. The second step consists of a tracking algorithm that aims at identifying patterns that resemble tree roots. In the last step, a continuous function is fitted to each root in order to effectively interpolate between points and subsequently estimate the density of the roots. This paper concludes with a case study on an urban tree at the Gunnersbury Park, London, United Kingdom. The top soil around the tree was excavated to $40 \mathrm{~cm}$ below the surface approximately in order to expose the tree root architecture. Then the exposed tree root system was used to compare the survey results for validity purposes and ultimately support the viability of the proposed data processing methods adopted in this investigation.
\end{abstract}

Keywords-Assessment of Tree Roots; Ground Penetrating Radar (GPR); Tree Health Monitoring; Tree Root Mass Density.

\section{INTRODUCTION}

The root system is the most crucial part of a tree since it is responsible for transporting water and minerals, vital for the well-being and longevity of the tree $[1,2]$. Besides, the root system protects the tree from the wind by providing it with stability and structural integrity [3]. Tree roots follow stochastic patterns that greatly vary amongst tree species and environmental conditions $[4,5]$. Moreover, tree patterns can also relate to the health status of the tree and have been used effectively as a diagnostic tool in arboriculture applications [6]. Lastly, as it is stated in [7], root biomass can be used as a robust indicator of $\mathrm{CO} 2$ concentration and carbon dynamics in general.

Various methodologies have been suggested in order to map the root structure of a given tree effectively. These approaches can be superficially divided into destructive and non-destructive testing (NDT) methods. Destructive methods include excavation, uprooting and profile wall technique [8]. These methods, apart from being unpractical and unsuitable for large scale forestry applications, they can also cause irreversible damage to the surrounding rhizosphere [8-10] and therefore are not preferred by foresters and tree officers. On the other hand, NDT methods can effectively map root patterns without disturbing the host material and without causing irreversible damage to the tree. Various NDT methods have been suggested for root mapping, from X-ray tomography, nuclear methods and magnetic resonance [11-13] to acoustic methods and electrical resistivity tomography [14]. Ground-penetrating radar is an NDT method with a unique wide span of applications that ranges from landmine detection [15] and archaeology [16] to glaciology and environmental applications [17]. GPR is a very appealing choice for forestry applications due to its versatility and high resolution. To that extend, GPR is starting to gain popularity amongst foresters and tree officers as an efficient tool for estimating root patterns in a non-destructive manner [18-20]. In the current paper, the GPR-based root-detection framework presented in $[21,22]$ is tested in a complete case study in a diseased urban tree within the greater London area in the United Kingdom. Concentric measurements were taken around the investigated tree, and the measured data were subjected to the processing pipeline presented in $[21,22]$. In particular, a threestep procedure was applied. The first step consists of a time-zero correction, zero-offset removal, time-varying gain and a singular value decomposition (SVD) filter. In the second step, a tracking algorithm is employed in order to connect points with high signal intensity within the processed B-Scans. In the third step, a continuous function is fitted to the points in order to interpolate between them and effectively estimate the density of the roots. After the completion of the measurements, the investigated area was excavated, and the hidden roots were accurately mapped for validation purposes. The resulting root structure using the processing pipeline described in $[21,22]$ was in good agreement with the actual roots indicating the potential of the proposed framework as an efficient forestry tool for large scale forestry applications. 


\section{AIM \& OBJECTIVES}

This research aims to demonstrate the capability of midrange frequency GPR antenna systems in efficiently localising tree roots and reconstructing the architecture of tree root systems.

To achieve this aim, the main objective of this research is to provide root density maps at different depths, to facilitate the interpretation of local variations of root concentration. Another essential objective is to prove the validity of the proposed methodology by way of comparison between the results the realtruth evidence following the excavation of the soil in the area previously investigated with the GPR.

\section{METHODOLOGY}

The proposed methodology is divided into three main data processing stages, as exhaustively described in [21, 22]. A preprocessing algorithm is applied, in which advanced signal processing techniques were employed. This stage aims to remove unwanted signal information and to increase the overall signal-to-noise ratio (SNR). Moreover, reflections from deeply localised targets are amplified, to reconstruct the overall architecture of the tree root system.

A multi-stage algorithm for the identification of roots and their tracking in a three-dimensional environment is applied afterwards. In the third phase, tree root mass density at different depths is estimated, based on the outcomes of the previous stage.

\section{A. Signal processing}

The primary purpose of this stage is to reduce noise-related information from the GPR data, as well as to achieve quantifiable information and easily interpretable images for the data analysis and interpretation.

To this extent, standard signal processing techniques are used in order to guarantee the broadest applicability of the proposed methodology. Then, the sequential use of a) zerooffset removal, b) zero correction, c) bandpass filtering and d) time-varying gain is carried out.

Nevertheless, GPR data can be heavily corrupted by ringing noise, a repetitive type of clutter with a high correlation between A-Scans which can lead to data misinterpretation. While the aforementioned techniques do not contribute to its removal, the SVD technique is known to be efficient in discarding the ringing noise contribution without affecting the original data. Therefore, the SVD filter is applied in this processing stage, to reduce the ringing noise and enhance the response of the targets.

\section{B. Tree Root Tracking Algorithm}

This stage of the methodology is formed by two main steps.

First, the fundamental hypotheses are initialised, that is, the data input settings (i.e. outcomes of the pre-processing algorithm, matrix dimensions, GPR data acquisition settings) and other important information (i.e. the data acquisition method and the dielectric properties of the medium).

Secondly, the pre-processed GPR data are analysed through an iterative procedure, so as to compare the amplitude values in different positions of the three-dimensional domain and find a correlation between them.

- Target detection: the amplitude values are compared with a predefined threshold value throughout the analysed domain. This step is necessary in order to identify the focused reflections that more likely belong to the roots of the tree.

- Correlation analysis: a possible correlation is sought between the targets identified in the previous step, based on their position in the three-dimensional space.

- $\quad$ Tracking of the roots: the correlated targets are joined into vectors that coincide with the spatial coordinates of the individual roots.

- Reconstruction of the root system architecture in the 3$\mathrm{D}$ domain: these vectors are positioned in a threedimensional space, thus creating a comprehensive rendering of the root system.

\section{Root Mass Density Estimation}

In the third and final phase, root mass density is estimated based on the results obtained in the previous step. Using a polynomial fitting, the path followed by the roots is better approximated in a continuous domain and, therefore, it is possible to calculate the length of each pattern. This led to the definition of an index for the calculation of the root mass density, which evaluates the length of the roots insisting on a reference volume $V$ :

$$
d=\frac{\sum_{i=1}^{n} L_{i}}{V}
$$

where $d$ is the density $\left[\mathrm{m} / \mathrm{m}^{3}\right], n$ is the number of roots contained in a reference unit of volume $\left[\mathrm{m}^{3}\right]$, and $L_{i}$ is the length of the root $[\mathrm{m}]$.

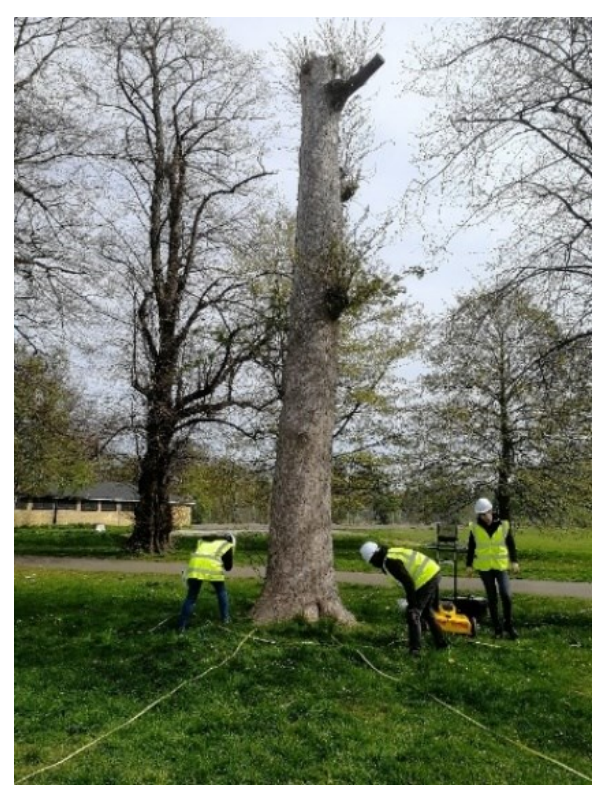

Fig. 1. The investigated area 


\section{CASE STUdy}

In this study, the viability of the proposed methodology was tested by investigating the soil around a tree in Gunnersbury Park, Ealing, London (United Kingdom). A semi-circular scanning configuration was employed for the creation of a threedimensional root system model and root density maps at different depths. Furthermore, an excavation survey was subsequently carried out for validation purposes in the same area.

\section{A. Data collection: the GPR Investigation}

A number of 36 semi-circular GPR scans were carried out around the investigated tree, starting $0.50 \mathrm{~m}$ from the bark and spaced $0.30 \mathrm{~m}$ from one another. Therefore, an overall area of $218.04 \mathrm{~m}^{2}$ was surveyed around the tree, with an outer radius of $11.86 \mathrm{~m}$ and an inner radius of $1.36 \mathrm{~m}$.

The survey was carried out using the Opera Duo groundcoupled GPR system, manufactured by IDS GeoRadar (part of Hexagon). The system is equipped with $700 \mathrm{MHz}$ and $250 \mathrm{MHz}$ central frequency antennas. Data were collected using a time window of $80 \mathrm{~ns}$, discretised across 512 samples. The horizontal resolution was set to $3.06 \times 10^{-2} \mathrm{~m}$. In order to achieve the optimal effective resolution, only data collected using the 700 $\mathrm{MHz}$ antenna were analysed.

\section{B. The Data Processing Framework: Main Results and Short Discussion}

The use of a pre-processing phase on GPR data allowed for an effective reduction of noise and a significant improvement in target detection. In more detail, the use of the SVD filter proved to be effective in removing horizontal layers and repeated reflections. This is relevant as the presence of these reflections could have affected the effectiveness of the subsequent steps of the algorithm. Fig. 2 shows a comparison between B-scans before (a) and after (b) the application of the pre-processing phase, proving how noise-related features are significantly attenuated or eliminated.

The study of the B-scans shows the presence of a buried structure (Fig. 2). To this effect, $\mathrm{C}$-scans were created to analyse size and configuration of this feature. As it is shown in Fig. 3, the study of the C-scans revealed the presence of a structure crossing the investigation area, most likely a conduit serving the artificial lake not far away in the park.
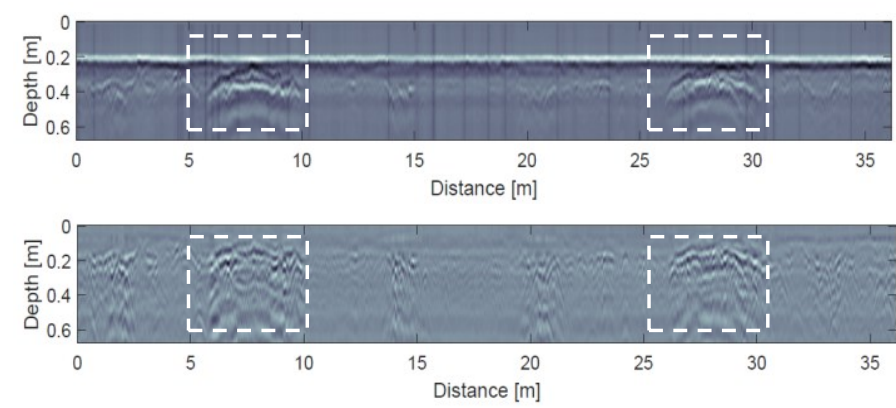

Fig. 2. An example of GPR data pre-processing. a) B-scan before the signal processing, b) B-scan after the signal processing. The presence of a buried structure is highlighted by the white dashed squares

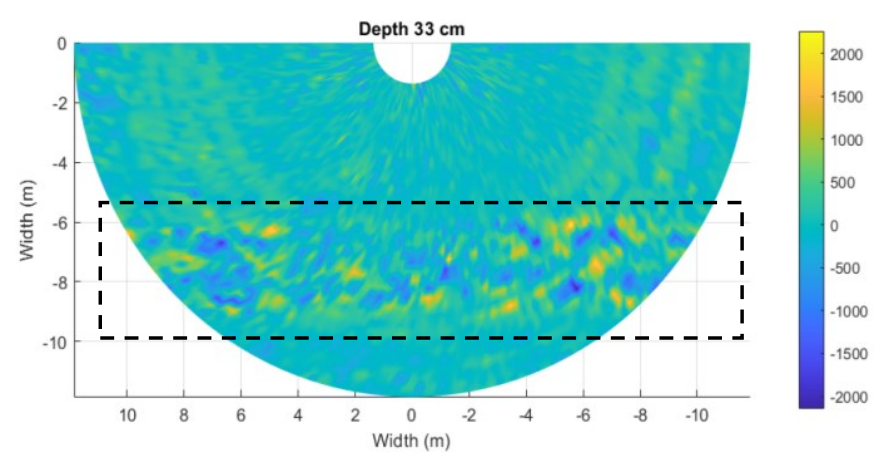

Fig. 3. A C-scan of the investigated area showing the presence of a buried structure

Subsequently, the algorithm for the analysis of the spatial correlation and the automatic tracking of the roots in the threedimensional space was applied to the processed data. Fig. 4 shows a two-dimensional aerial view (a) and a threedimensional rendering of the investigated area. In order to make the result interpretation easier, the roots in the shallow subsoil (i.e., the first $0.25 \mathrm{~m}$ of soil) are highlighted in green, whereas deeper roots are highlighted in brown. The result obtained clearly demonstrates the ability of the proposed algorithm to provide a detailed and accurate reconstruction of the root system. For sake of consistency, the point coordinates were double-checked against the positions of the focused targets, through the analysis of the single B-scans.
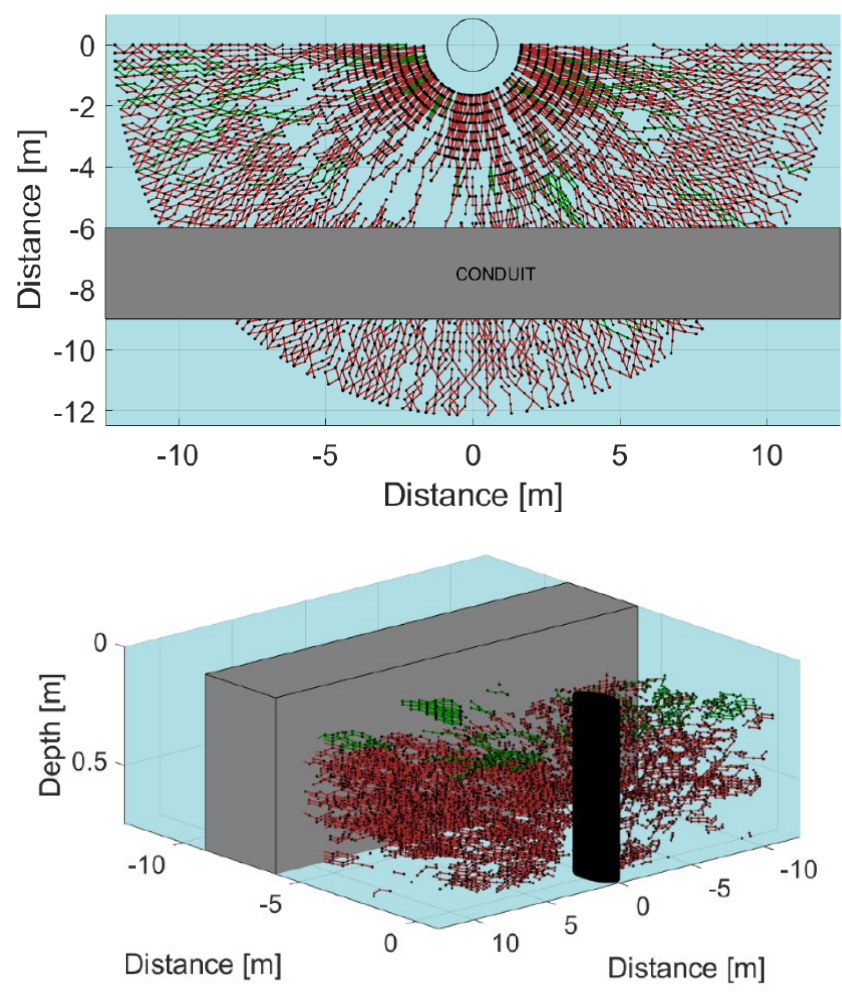

Fig. 4. Reconstruction of the root system architecture of the surveyed tree: a) $2 \mathrm{D}$ aerial view and b) 3D reconstruction 


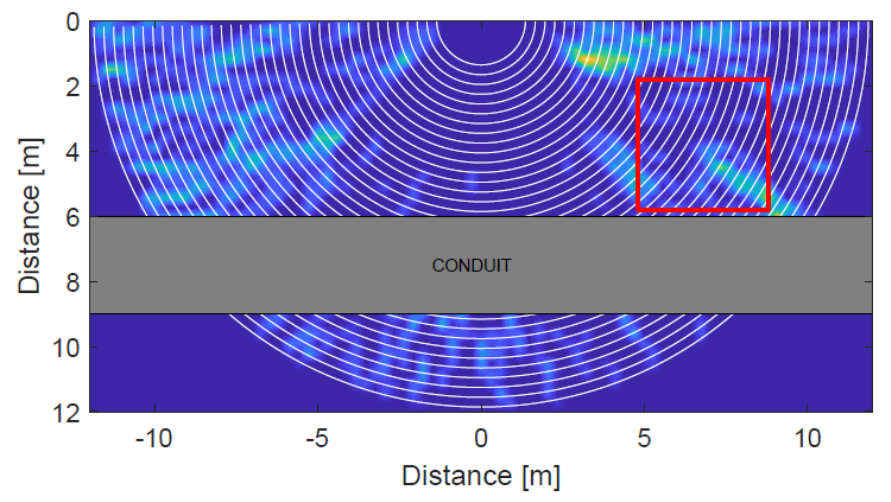

Fig. 5. Root mass density map, related to a depth from $0.20 \mathrm{~m}$ to $0.30 \mathrm{~m}$

At the final stage, the root mass density at different depths was investigated by means of the proposed index. The investigated domain was divided into reference volumes $(0.3 \mathrm{~m}$ $\times 0.3 \mathrm{~m} \times 0.1 \mathrm{~m})$ and then analysed to evaluate the total length of roots per reference unit. Fig. 5 presents an example of the outcomes of this stage of analysis, in the form of a density map related to a depth range from $0.50 \mathrm{~m}$ to $0.60 \mathrm{~m}$.

\section{The Validation Process}

An area of $4 \mathrm{~m}$ per side (highlighted by the red square in Fig. 5) was selected for validation purposes and it was excavated. The position of the excavation area was identified precisely, based on the coordinates of the GPR survey previously carried out (Fig. 6). The excavation was carried out by removing a layer of $0.10 \mathrm{~m}$ of soil at a time, so as to be consistent with the depth scale of the density maps produced.

The excavation revealed the presence of several roots, as shown in Fig. 6. Position and depth of roots were consistent with those reported in the density maps. As an example, a cluster of roots was found at the top-left corner of the excavation area at a depth between approximately $0.20 \mathrm{~m}$ and $0.25 \mathrm{~m}$, as shown in Fig. 7. This is coherent with the indications provided by the concerning mass density map (see Fig. 5), which indicates a relatively small density of roots within this area.

In addition, the presence of several boulders was noted during the excavation process, with a main size of about $0.1 \mathrm{~m}$ (Fig. 8). Their location was compared with the outcomes of the root density maps, and no evidence was found.

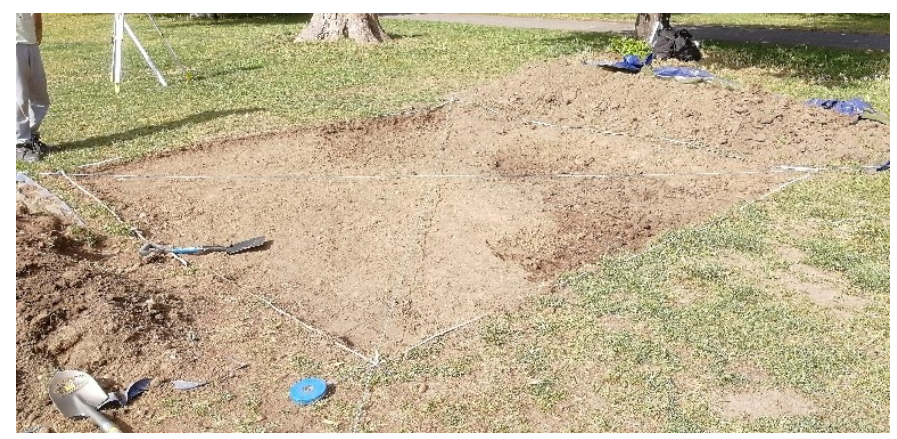

Fig. 6. Overview of the excavation area

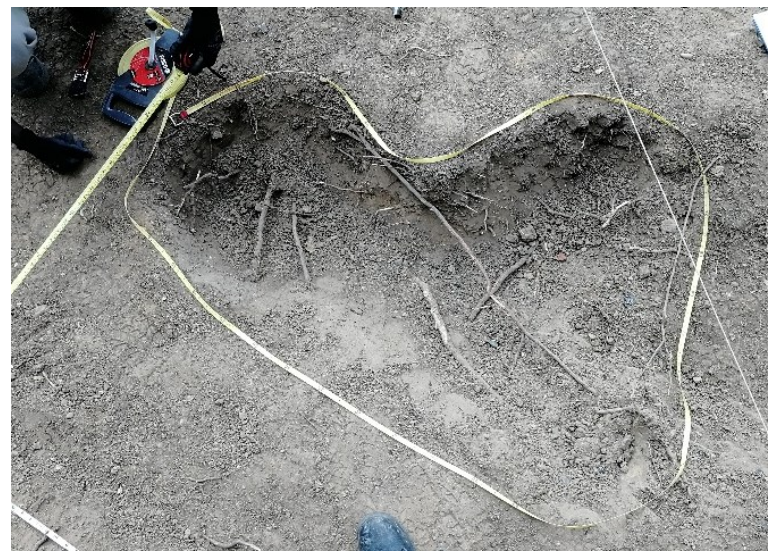

Fig. 7. Detail of the excavation

This supports the validity of the proposed methodology, as the algorithm has proven to be able to discard false alarms, i.e. uncoherent signal reflections not related to tree roots.

\section{CONCLUSION}

This study reports on an investigation within the context of the applications of ground penetrating radar (GPR) in detecting and establishing the tree roots system and also producing a 3 dimensional depiction of the tree root architecture using a set of novel data processing techniques. To realise this, a multi-stage interpretation algorithm was introduced, in order to reconstruct the tree root patterns based on the collected data. The proposed methodology is based on the utilisation of a set of semi-circular scans, which expand outwards radially starting from the trunk of the tree. Initially, a signal processing stage was applied, to remove noise-related information and enhance the response from the real targets. Subsequently, a tracking algorithm was used in order to locate and automatically track viable root paths. Lastly, the identified roots were expressed through continuous functions, in order to analytically derive the root mass density. A case study is presented, in which the proposed method was successfully applied. The tracking algorithm has proven able to identify both the shallow (i.e. within the first $25 \mathrm{~cm}$ of soil) and deep (i.e. below $25 \mathrm{~cm}$ from the surface of the soil) root structures.

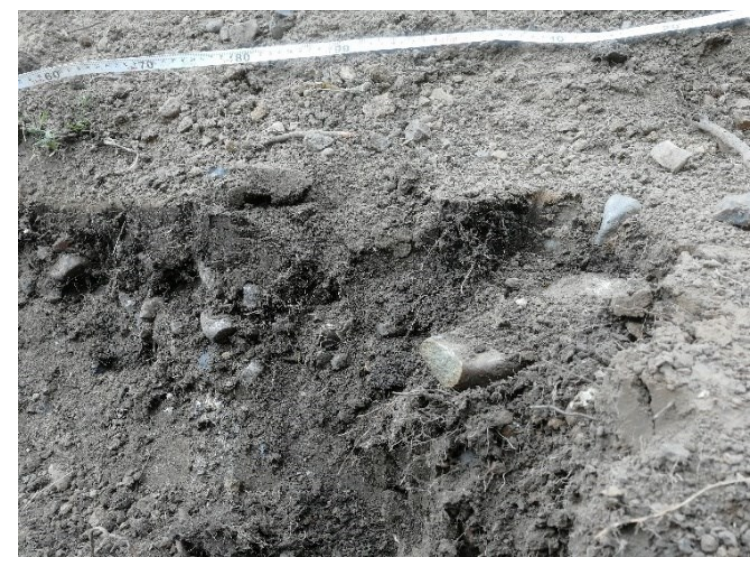

Fig. 8. Detail of the presence of boulders at the top-left corner of the excavation area 
Based on this result, root mass density maps at different depths were estkimagted. To prove the validity of the proposed methodology, a validation survey was carried out, in which a part of the previously investigated area was excavated and tree roots were exposed. The density maps were in good agreement with the actual root structure, whereas the presence of boulders of appreciable size was not detected. This confirms the viability of the proposed methodology and supports the premise that GPR is a powerful NDT tool for the assessment of tree roots in large scale forestry applications.

\section{ACKNOWLEDGEMENTS}

The authors would like to express their sincere thanks and gratitude to the following trusts, charities, organisations and individuals for their generosity in supporting this project: Lord Faringdon Charitable Trust, The Schroder Foundation, Cazenove Charitable Trust, Ernest Cook Trust, Sir Henry Keswick, Ian Bond, P. F. Charitable Trust, Prospect Investment Management Limited, The Adrian Swire Charitable Trust, The John Swire 1989 Charitable Trust, The Sackler Trust, The Tanlaw Foundation, and The Wyfold Charitable Trust. This paper is dedicated to the memory of Jonathan West, a friend, a colleague, a forester, a conservationist and an environmentalist who died following an accident in the woodland that he loved.

\section{REFERENCES}

[1] A. Eshel and T. Beeckman, Plant Roots: The Hidden Half, CRC Press, 2013.

[2] J.D. Deans, "Dynamics of Coarse Root Production in a Young Plantation of Picea sitchensis," Forestry (Lond), vol. 54, pp. 139-155, /01/01. 1981.

[3] S.G. Pallardy, Physiology of woody plants, Academic Press, 2010.

[4] B.F. Wilson, Structure and growth of woody roots of Acer rubrum L, Harvard University, Harvard Forest St. Petersham, 1964.

[5] A. Stokes, A.H. Fitter and M.P. Courts, "Responses of young trees to wind and shading: effects on root architecture," J.Exp.Bot., vol. 46, pp. 11391146, 1995.

[6] J.L. Innes, Forest health: its assessment and status. $\mathrm{CAB}$ international, 1993.

[7] D.B. Stover, F.P. Day, J.R. Butnor and B.G. Drake, "Effect of elevated $\mathrm{CO} 2$ on coarse-root biomass in Florida scrub detected by groundpenetrating radar," Ecology, vol. 88, pp. 1328-1334, 2007.
[8] B. Reubens, J. Poesen, F. Danjon, G. Geudens and B. Muys, "The role of fine and coarse roots in shallow slope stability and soil erosion control with a focus on root system architecture: a review," Trees, vol. 21, pp. 385-402, 2007.

[9] L. Guo, J. Chen, X. Cui, B. Fan and H. Lin, "Application of ground penetrating radar for coarse root detection and quantification: a review," Plant Soil, vol. 362, pp. 1-23, 2013.

[10] J. Čermák, N. Nadezhdina, L. Meiresonne and R. Ceulemans, "Scots pine root distribution derived from radial sap flow patterns in stems of large leaning trees," Plant Soil, vol. 305, pp. 61-75, 2008.

[11] P.J. Gregory, D.J. Hutchison, D.B. Read, P.M. Jenneson, W.B. Gilboy and E.J. Morton, "Non-invasive imaging of roots with high resolution Xray micro-tomography," in Roots: the dynamic interface between plants and the Earth, Springer, 2003, pp. 351-359.

[12] C.J. Moran, A. Pierret and A.W. Stevenson, "X-ray absorption and phase contrast imaging to study the interplay between plant roots and soil structure," Plant Soil, vol. 223, pp. 101-117, 2000.

[13] C.M. Paglis, "Application of electrical resistivity tomography for detecting root biomass in coffee trees," International Journal of Geophysics, vol. 2013, 2013.

[14] M. Amato, B. Basso, G. Celano, G. Bitella, G. Morelli and R. Rossi, "In situ detection of tree root distribution and biomass by multi-electrode resistivity imaging," Tree Physiol., vol. 28, pp. 1441-1448, 2008.

[15] I. Giannakis, A. Giannopoulos and C. Warren, "A realistic FDTD numerical modeling framework of ground penetrating radar for landmine detection," IEEE Journal of Selected Topics in Applied Earth Observations and Remote Sensing, vol. 9, pp. 37-51, 2015.

[16] D.J. Daniels, Ground Penetrating Radar, IET, 2004.

[17] F. Tosti, L.B. Ciampoli, F. D'Amico, A.M. Alani and A. Benedetto, "An experimental-based model for the assessment of the mechanical properties of road pavements using ground-penetrating radar," Constr.Build.Mater., vol. 165, pp. 966-974, 2018.

[18] J. Hruska, J. Čermák and S. Šustek, "Mapping tree root systems with ground-penetrating radar," Tree Physiol., vol. 19, pp. 125-130, 1999.

[19] C.V. Barton and K.D. Montagu, "Detection of tree roots and determination of root diameters by ground penetrating radar under optimal conditions," Tree Physiol., vol. 24, pp. 1323-1331, 2004.

[20] J.R. Butnor, J.A. Doolittle, K.H. Johnsen, L. Samuelson, T. Stokes and L Kress, "Utility of ground-penetrating radar as a root biomass survey tool in forest systems," Soil Sci.Soc.Am.J., vol. 67, pp. 1607-1615, 2003.

[21] A.M. Alani and L. Lantini, "Recent advances in tree root mapping and assessment using non-destructive testing methods: a focus on ground penetrating radar," Surv.Geophys., pp. 1-42, 2019.

[22] L. Lantini, F. Tosti, I. Giannakis, D. Egyir, A. Benedetto and A.M. Alani, "A novel processing framework for tree root mapping and density estimation using ground penetrating radar," in 10th International Workshop on Advanced Ground Penetrating Radar, pp. 1-6, 2019. 